\title{
3D Ultrasound to Stereoscopic Camera Registration through an Air-Tissue Boundary
}

\author{
Michael C. Yip ${ }^{1}$, Troy K. Adebar ${ }^{1}$, Robert N. Rohling ${ }^{1}$, \\ Septimiu E. Salcudean ${ }^{1}$, and Christopher Y. Nguan $^{2}$ \\ 1 University of British Columbia, British Columbia V6T1Z4 Canada \\ 2 Vancouver General Hospital, Vancouver, British Columbia V5Z1M9 Canada
}

\begin{abstract}
A novel registration method between 3D ultrasound and stereoscopic cameras is proposed based on tracking a registration tool featuring both ultrasound fiducials and optical markers. The registration tool is pressed against an air-tissue boundary where it can be seen both in ultrasound and in the camera view. By localizing the fiducials in the ultrasound volume, knowing the registration tool geometry, and tracking the tool with the cameras, a registration is found. This method eliminates the need for external tracking, requires minimal setup, and may be suitable for a range of minimally invasive surgeries. A study of the appearance of ultrasound fiducials on an air-tissue boundary is presented, and an initial assessment of the ability to localize the fiducials in ultrasound with sub-millimeter accuracy is provided. The overall accuracy of registration $(1.69 \pm 0.60 \mathrm{~mm})$ is a noticeable improvement over other reported methods and warrants patient studies.
\end{abstract}

\section{Introduction}

Augmented reality in surgery often involves the superposition of medical images of a patient's anatomy onto a camera-based image of the overlying tissues. With sufficient registration accuracy, the surgeon is then able to localize the internal anatomy (subsurface features such as lesions or nerves) for improved surgical guidance. Prior work in augmented reality for surgical applications has been applied to x-ray, computed tomography (CT), magnetic resonance imaging (MRI) and ultrasound (US) [1]-7]. This paper explores the ability to display an external three-dimensional ultrasound (3DUS) volume in a laparoscopic camera view during minimally invasive surgery.

To date, registration between an US volume and camera images has generally involved two tasks: calibrating the ultrasound volume to the pose of the US transducer, and tracking both the US transducer and the cameras in 3D space. For the first task, numerous techniques for US transducer calibration have been previously investigated; Lindseth [8] and Mercier [9] offer extensive reviews on the subject of US transducer calibration. The second task, tracking of the cameras and an US transducer, can be performed using magnetic trackers, optical trackers, or robot kinematics [5]- 7]. While these techniques have proven useful in the operating room, they still have their shortcomings: time-consuming transducer

T. Jiang et al. (Eds.): MICCAI 2010, Part II, LNCS 6362, pp. 626 634, 2010.

(C) Springer-Verlag Berlin Heidelberg 2010 
calibrations, additional tracking equipment, line-of-sight issues, modifications to the US transducers and cameras for tracking, and consequently the consumption of valuable time and space in the operating room. In addition, cumulative errors in transducer calibration and equipment tracking contribute to errors in registration that may be amplified by a lever-arm effect.

We address the above issues by introducing a new technique for registering stereoscopic cameras and 3DUS using a registration tool. Fiducials attached to the registration tool are held against the air-tissue boundary and imaged through the tissue. Their locations in the US volume are determined, and through the known geometry of the tool and the tracking of the tool by the stereoscopic cameras, a 3DUS to camera registration can be found. This provides a direct transformation from the US to the stereoscopic cameras, thus eliminating errors related to calibrating the US transducer and tracking the US transducer and the cameras. Registration of 3DUS to the cameras directly across the air-tissue boundary is the key innovation of this paper.

We envisage using the novel registration system in laparoscopic surgery, where structures being operated on can be imaged more effectively with external or endocavity US, or with laparoscopic US from a different direction than the camera view. For example, during partial nephrectomy, the kidney can be imaged with external US through the abdomen, or with laparoscopic US from one side of the kidney while the camera and laparoscopic instruments operate from the other. Similarly, in laparoscopic radical prostatectomy, endorectal US can image the prostate as in prostate brachytherapy, while the surgery proceeds with the trans-abdominal or trans-perineal approach. In both cases, registration is required to overlay the US image onto the endoscopic camera view. We propose that it be carried out with the technique described in this paper.

\section{Methods}

Our study had two goals: to determine the accuracy of locating US fiducials on an air-tissue boundary, and to determine the feasibility of using these fiducials to register 3DUS to stereoscopic cameras. We first examined the accuracy of localizing spherical fiducials on an air-tissue boundary in US. Air-tissue boundaries exhibit high reflection at their surfaces that may make it difficult to accurately localize fiducials. We considered five variables that could affect the accuracy of fiducial localization: (1) fiducial size, (2) lateral position in US image, (3) angle of air-tissue boundary, (4) boundary depth, and (5) stiffness of tissue.

Next, we implemented a direct closed-form registration method between 3DUS and a stereoscopic camera by localizing surface fiducials in both the 3DUS volume and the stereo camera (Figure 1 $\mathrm{b}$ ). This method is described below.

We begin by defining four coordinate systems, the stereo camera system $\left\{\underline{o}_{0}, \underline{C}_{0}\right\}$, the optical marker system $\left\{\underline{Q}_{1}, \underline{C}_{1}\right\}$, the US fiducial system $\left\{\underline{o}_{2}, \underline{C}_{2}\right\}$ and the 3DUS system $\left\{\underline{o}_{3}, \underline{C}_{3}\right\}$. The transformation from $\left\{o_{1}, \underline{C}_{1}\right\}$ to $\left\{\underline{o}_{0}, \underline{C}_{0}\right\}$, ${ }^{0} T_{1}$, is found by stereo-triangulating the optical markers on the registration tool. The transformation from $\left\{\alpha_{2}, \underline{C}_{2}\right\}$ to $\left\{\underline{o}_{1}, \underline{C}_{1}\right\},{ }^{1} T_{2}$, is constant and known from 
the tool geometry. The transformation from $\left\{\underline{Q}_{3}, \underline{C}_{3}\right\}$ to $\left\{\underline{Q}_{2}, \underline{C}_{2}\right\},{ }^{2} T_{3}$, is found by localizing three fiducials that define $\left\{\underline{Q}_{2}, \underline{C}_{2}\right\}$ in the 3DUS system $\left\{\underline{Q}_{3}, \underline{C}_{3}\right\}$.

The three fiducial locations in coordinate system $\left\{\underline{Q}_{3}, \underline{C}_{3}\right\},{ }^{3} x_{0},{ }^{3} x_{1}$ and ${ }^{3} x_{2}$, define two perpendicular vectors with coordinates

$$
\begin{aligned}
& { }^{3} v_{1}={ }^{3} x_{1}-{ }^{3} x_{0} \\
& { }^{3} v_{2}={ }^{3} x_{2}-{ }^{3} x_{0},
\end{aligned}
$$

that can be used to define the unit vectors of frame $\underline{C}_{2}$ in system $\left\{\alpha_{3}, \underline{C}_{3}\right\}$ :

$$
\begin{aligned}
{ }^{3} i_{2} & =\frac{{ }^{3} v_{1}}{\left\|{ }^{3} v_{1}\right\|} \\
{ }^{3} k_{2} & =\frac{{ }^{3} v_{1} \times{ }^{3} v_{2}}{\left\|{ }^{3} v_{1} \times{ }^{3} v_{2}\right\|} \\
{ }^{3} j_{2} & ={ }^{3} k_{2} \times{ }^{3} i_{2} .
\end{aligned}
$$

The origin $Q_{2}$ has coordinates ${ }^{3} x_{0}$ in $\left\{\underline{o}_{3}, \underline{C}_{3}\right\}$. The homogeneous transformation from the 3DUS system $\left\{o_{3}, \underline{C}_{3}\right\}$ to the US fiducial system $\left\{\alpha_{2}, \underline{C}_{2}\right\},{ }^{3} T_{2}$, is then

$$
{ }^{3} T_{2}=\left[\begin{array}{cccc}
{ }^{3} i_{2} & { }^{3} j_{2} & { }^{3} k_{2} & { }^{3} x_{0} \\
0 & 0 & 0 & 1
\end{array}\right]
$$

and ${ }^{2} T_{3}={ }^{3} T_{2}{ }^{-1}$. The overall transformation between the stereo camera system $\left\{o_{0}, \underline{C}_{0}\right\}$ and the 3DUS system $\left\{\underline{o}_{3}, \underline{C}_{3}\right\}$ is then

$$
{ }^{0} T_{3}={ }^{0} T_{1}{ }^{1} T_{2}{ }^{2} T_{3} .
$$

A homogenous transformation can then be constructed to register the 3DUS frame to the stereo camera frame. Lastly, with known camera parameters (focal length, image center, distortion coefficient, etc.), the registered US volume in the camera frame can be projected onto the two stereoscopic images.

\subsection{Experimental Setup}

Figure 1b shows the experimental setup used in this study. 3DUS volumes were captured using a Sonix RP US machine (Ultrasonix Medical Corp., Richmond, Canada) with a mechanical 3D transducer (model 4DC7-3/40). A three-axis mechanical micrometer stage was used for accurate positioning of registration tools relative to the fixed US transducer and stereo cameras.

Surface Fiducial Localization. Sets of steel spherical fiducials arranged in precisely known geometry were pressed against tissue-mimicking phantoms, imaged and localized in the 3DUS volumes. The steel plates contained three sets of fiducials spaced $10 \mathrm{~cm}$ apart, with each set consisting of a center fiducial and eight surrounding fiducials at a radius of $10 \mathrm{~mm}$ (Figure 2 2 ). The fiducials were seated in holes cut into the plate on a water jet cutter with dimensional accuracy 


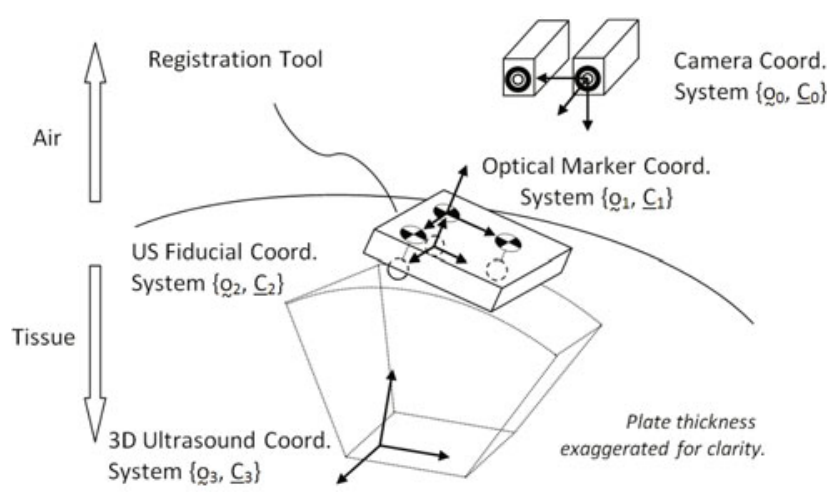

(a)

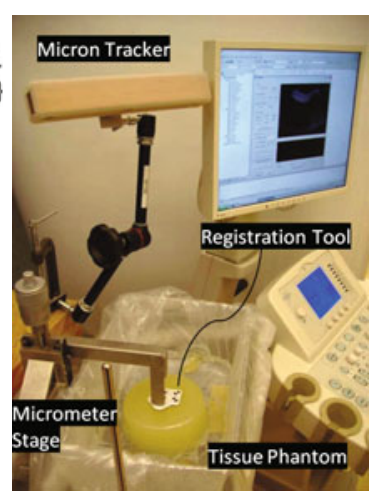

(b)

Fig. 1. a) Schematic of the registration method, b) Experimental Setup

of $0.13 \mathrm{~mm}$. Fiducial diameters of $2 \mathrm{~mm}, 3 \mathrm{~mm}$ and $4 \mathrm{~mm}$ were imaged through phantoms with thicknesses of $3 \mathrm{~cm}, 6 \mathrm{~cm}$ and $9 \mathrm{~cm}$, stiffnesses of $12 \mathrm{kPa}, 21 \mathrm{kPa}$ and $56 \mathrm{kPa}$, and boundary angles of 0 degrees, 20 degrees and 40 degrees. The phantoms were made from polyvinyl chloride (PVC) using a ratio of liquid plastic to softener of 1:1 (12 kPa, low stiffness), 2:1 (21 kPa, medium stiffness), and :0 (56 kPa, high stiffness) to create phantoms that mimicked tissue properties [10]. To create fully developed speckle, one percent (mass) cellulose was added as a scattering agent.

The five independent variables evaluated were varied independently about a control case $(3 \mathrm{~mm}$ fiducials, $6 \mathrm{~cm}$ depth, $21 \mathrm{kPa}$ stiffness, 0 degree angle, and central location). The surface fiducial plates were pressed lightly into the PVC tissue phantoms, and imaged through the phantoms. The fiducials were then manually localized in the US volume, and the Euclidean distances between the outer fiducials and the center fiducials were compared to the known geometry to determine the accuracy of localization. For every variable level, 10 tests with 8 error measurements were performed $(n=80)$. The focal depth was set to the boundary depth in all tests.

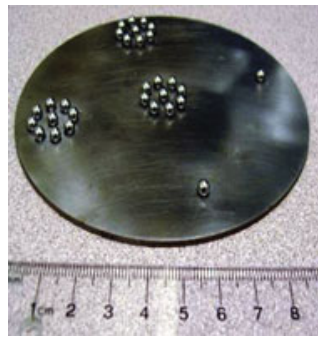

(a)

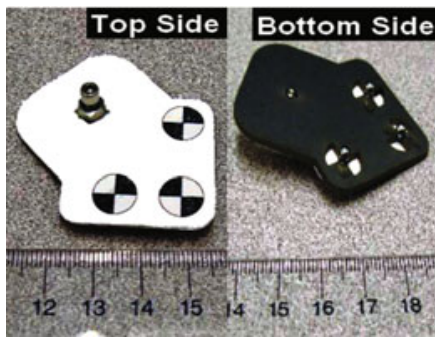

(b)

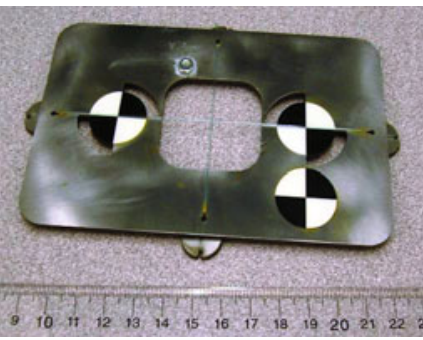

(c)

Fig. 2. a) Fiducial localization test plate, b) Registration tool, c) Registration accuracy test tool 
Registration. For the registration experiments, a Micron Tracker H3-60 optical tracking system (Claron Technology, Toronto, Canada) was used as the stereoscopic cameras. This provided a stable and accurate pre-calibrated camera system and allowed the analysis of registration accuracy to focus mainly on localizing the registration tool in US. The registration tool was also built on a steel plate cut on a waterjet cutter (Figure 2 $\mathrm{b}$ ). On the top surface are three Micron Tracker markers spaced $20 \mathrm{~mm}$ and $15 \mathrm{~mm}$ apart forming an "L" shape; on the bottom surface are three surface fiducials $(3 \mathrm{~mm})$ seated in holes cut directly in line with the Micron Tracker markers. Registration accuracy was measured using the test tool shown in Figure 2r. The test tool consists of a steel frame, a nylon wire cross which can be accurately localized by US in a water bath, and three Micron Tracker markers which allow the tracker to determine the location of the wire cross in the camera frame.

We first determined the homogeneous transformation relating points in the US frame to the camera frame using the registration tool. We then evaluated the accuracy of the transformation using the test tool placed in a water bath. The crosswire location in US registered into the camera frame was compared to the location of the crosswire in the camera coordinates. This was done by saving a US volume of the crosswire in a water bath, and then draining the water to track the optical markers on the test tool in the stereoscopic cameras. Registration error was defined as the Euclidean distance between the position predicted by registration and the tracked position of the crosswire in the cameras. The errors were transformed into the US frame so that they could be specified in the lateral, elevational and axial directions. To ensure accurate scales, the US volumes were generated using the correct speeds of sound for the phantoms and for water.

\section{Results}

The results of the fiducial localization experiments are shown in Table 1 . Hypothesis testing was used to determine the statistical signficance of variables. Given two groups $x$ and $y$, unpaired student $t$-tests determine the probability $p$ that the null hypothesis $\left(\mu_{x}=\mu_{y}\right)$ was true. A one-way analysis of variance (ANOVA) is a generalization of the student $t$-test for more than two groups and produces probability statistics $\{F, p\}$. For both the student $t$-test and ANOVA, a generally accepted probability for suggesting statistical significance is $p<0.05$.

The ANOVA results showed that the size of the fiducial, the depth of the boundary, and the angle at which the boundary was imaged affect the accuracy of fiducial localization $\left(\right.$ ANOVA: $F_{\text {size }}=7.34, p_{\text {size }}=9.96 E-04 ; F_{\text {depth }}=$ $\left.15.5, p_{\text {depth }}=1.11 E-06 ; F_{\text {angle }}=8.49, p_{\text {angle }}=3.61 E-04\right)$. However, the tissue stiffness does not significantly change the accuracy of fiducial localization (ANOVA: $\left.F_{\text {stiffness }}=0.0414, p_{\text {stiffness }}=0.960\right) . T$-tests results showed that the lateral position of the fiducials on the boundary plays a significant role in the accuracy of localization (t-test: $p=7.10 E-18$ ).

In our registration experiment, four unique poses of the registration tool were used for a physical configuration of the camera and US transducer. The registration errors were computed at twelve different locations in the US volume. To test 
Table 1. Mean, standard deviation and median of errors associated with localizing bead fiducials at air-tissue boundaries. Significantly different from control case $=\left(^{*}\right)$

\begin{tabular}{|c|c|c|c|c|}
\hline Variable & Value & Mean \pm Std Dev. $(\mathrm{mm})$ & Median $(\mathrm{mm})$ & RMS Error \\
\hline Fiducial Size & $2 \mathrm{~mm}$ & $0.94 \pm 0.34^{*}$ & 0.89 & 1.00 \\
& $3 \mathrm{~mm}$ & $0.82 \pm 0.28$ & 0.78 & 0.87 \\
& $4 \mathrm{~mm}$ & $0.70 \pm 0.20$ & 0.67 & 0.73 \\
\hline Boundary Depth & Long $(9 \mathrm{~cm})$ & $0.54 \pm 0.18^{*}$ & 0.55 & 0.57 \\
& Med. $(6 \mathrm{~cm})$ & $0.82 \pm 0.28$ & 0.78 & 0.87 \\
& Short $(3 \mathrm{~cm})$ & $0.66 \pm 0.20^{*}$ & 0.64 & 0.69 \\
\hline Tissue Stiffness & High $(12 \mathrm{kPa})$ & $0.81 \pm 0.30$ & 0.78 & 0.86 \\
& Med. $(21 \mathrm{kPa})$ & $0.82 \pm 0.28$ & 0.78 & 0.87 \\
& Low $(56 \mathrm{kPa})$ & $0.80 \pm 0.19$ & 0.80 & 0.82 \\
\hline Boundary Angle & $0^{\circ}$ & $0.82 \pm 0.28$ & 0.78 & 0.87 \\
& $20^{\circ}$ & $0.78 \pm 0.28$ & 0.75 & 0.83 \\
& $40^{\circ}$ & $1.04 \pm 0.35^{*}$ & 0.97 & 1.10 \\
\hline Lateral Position & Center & $0.82 \pm 0.28^{*}$ & 0.78 & 0.87 \\
On Boundary & Offset $(10 \mathrm{~cm})$ & $0.60 \pm 0.28^{*}$ & 0.59 & 0.66 \\
\hline
\end{tabular}

Table 2. Mean errors $(n=12)$ between points in a registered 3DUS volume and its location in the stereo-camera frame

\begin{tabular}{|l|c|c|c|c|}
\hline & $e_{\text {Lateral }}(\mathrm{mm})$ & $e_{\text {Elevational }}(\mathrm{mm})$ & $e_{\text {Axial }}(\mathrm{mm})$ & $e_{\text {Total }}(\mathrm{mm})$ \\
\hline Registration 1 & $0.90 \pm 0.44$ & $0.77 \pm 0.33$ & $1.08 \pm 0.75$ & $1.75 \pm 0.56$ \\
Registration 2 & $1.02 \pm 0.45$ & $0.60 \pm 0.32$ & $1.14 \pm 0.99$ & $1.83 \pm 0.74$ \\
Registration 3 & $0.65 \pm 0.43$ & $0.76 \pm 0.33$ & $1.01 \pm 0.63$ & $1.55 \pm 0.53$ \\
Registration 4 & $0.57 \pm 0.40$ & $0.82 \pm 0.30$ & $1.03 \pm 0.79$ & $1.60 \pm 0.58$ \\
\hline Average & $0.78 \pm 0.45 \mathrm{~mm}$ & $0.74 \pm 0.32 \mathrm{~mm}$ & $1.07 \pm 0.78 \mathrm{~mm}$ & $1.69 \pm 0.60 \mathrm{~mm}$ \\
\hline
\end{tabular}

the repeatability of this method, the registration was repeated four times on the same images. Table 2 shows that the average error among all the transformed points for all transformations was $1.69 \mathrm{~mm}$, with a minimum error of $1.55 \mathrm{~mm}$ and a maximum error of $1.84 \mathrm{~mm}$. The time required to perform a registration was approximately equal to the acquisition time of a 3DUS volume (2 sec).

\section{Discussion}

The fiducial localization tests showed that errors associated with localizing surface fiducials at an air-tissue boundary ranged from $0.54 \mathrm{~mm}$ to $1.04 \mathrm{~mm}$. Several variables had a significant effect on accuracy. The smaller fiducials $(2 \mathrm{~mm})$ produced higher localization errors, suggesting that the fiducials became lost in the boundary reflection. The larger fiducials presented larger features that were easier to detect. Boundary depths farther away from the US transducer produced lower localization errors, as fiducial centers were more difficult to localize when approaching the near field [11]. 
Two results from the localization error analysis that have practical implications are that tissue stiffness does not significantly affect the accuracy of fiducial localization and that only large angles (e.g. 40 degrees) significantly affect the localization accuracy. Our registration method should therefore remain accurate for tissues with a wide variety of stiffnesses and shapes. The lateral location of the fiducials on the air-tissue boundary, however, was significant to the localization accuracy. The air-tissue boundary exhibited greater specular reflection near the axis of the US transducer, and thus fiducials offset laterally from the axis were less obscured by specular reflection and could be more accurately localized.

The registration experiment showed that using fiducials on an air-tissue boundary for direct registration between 3DUS and stereo cameras is feasible with an accuracy of $1.69 \pm 0.60 \mathrm{~mm}$. The largest errors were in the axial directions since the tail artifacts of the surface fiducials obscured the true depth at which the fiducials were located in the US volume (Figure 3). Repeated registrations on the same data and registrations using different physical locations of the registration tool all gave consistent overall and component errors, suggesting a model of the reverberation tail could improve localization and registration accuracy further. Nevertheless, based on the overall errors, our registration method is a promising alternative to using tracking equipment, where errors for similar US-to-camera registration systems are within $3.05 \pm 0.75 \mathrm{~mm}[5]$ for magnetic tracking and $2.83 \pm 0.83 \mathrm{~mm}[6$ ] for optical tracking. It is clear that the main source of error for the new registration method is the localization of registration tool fiducials, as any localization errors would be amplified by a lever-arm effect.
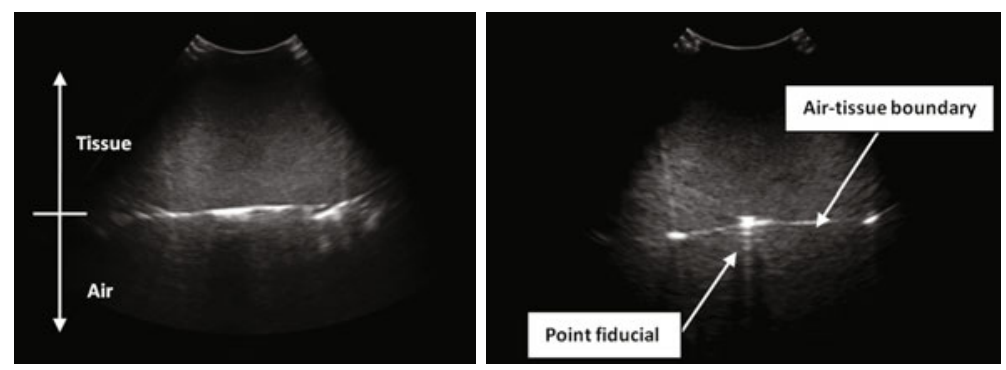

Fig. 3. Example images of an air-tissue boundary (left) and a $3 \mathrm{~mm}$ fiducial pressed against an air-tissue boundary (right)

The proposed registration is ideal for situations where the camera and the US transducer are fixed. However, if the US transducer or the camera is moved, a new registration can simply be acquired. Alternatively, in the case of robot-assisted surgery, the robot kinematics can be used to determine the new locations of the camera or the US transducer and maintain continuous registration to within the accuracy of robot kinematic calculations from joint angle readings.

A few practical issues with the proposed registration method should be considered. First, stereo-camera disparity plays a significant role in the accuracy 
of registration. The registrations presented in this paper were performed using the Claron Micron Tracker; this represents an ideal case, as the cameras have a large disparity $(12 \mathrm{~cm})$ and a tracking error of $\pm 0.2 \mathrm{~mm}$. In minimally invasive surgery, laparoscopic stereo-cameras having much smaller disparities would be used, possibly resulting in higher errors (although the cameras are imaging a much shallower depth so that the effect of disparity is lessened). This can be compensated for by maximizing the size of the registration tool, producing a well-conditioned system for computing the transformations. Such a registration tool could be designed to fold and fit through a trocar for laparoscopic surgery.

Another way to improve registration accuracy is to introduce redundancy into the registration data. Our registration tool featured only the minimum three fiducials required to extract the six degrees of freedom transformation between the US volume and the stereoscopic cameras; with more fiducials on the registration tool, averaging could be used to reduce errors. In addition, higher accuracies can be achieved by considering different poses of the registration tool in both the US and the camera frame [12].

\section{Conclusions}

In this study, we evaluated the accuracy of localizing fiducials pressed against an air-tissue boundary in ultrasound. We have shown that this method can be used to perform 3D ultrasound to stereo camera registration for augmented reality in surgery. This method provides a direct closed-form registration between a 3DUS volume and a stereoscopic camera view, does not require calibration of the US transducer or tracking of cameras or US transducers, and provides improved accuracies over tracking-based methods. Future work will utilize laparoscopic stereo-cameras in the registration technique, and investigate in-vivo studies to confirm an acceptable level of accuracy is achieved in an intra-operative setting.

Acknowledgments. The authors wish to thank NSERC and the CIHR Knowledge Translation Fund for supporting this work.

\section{References}

1. Grimson, E., Leventon, M.: Clinical experience with a high precision image-guided neurosurgery system. In: Wells, W.M., Colchester, A.C.F., Delp, S.L. (eds.) MICCAI 1998. LNCS, vol. 1496, pp. 63-73. Springer, Heidelberg (1998)

2. Fuchs, H., Livingston, M.A., Raskar, R., et al.: Augmented Reality Visualization for Laparoscopic Surgery. In: Wells, W.M., Colchester, A.C.F., Delp, S.L. (eds.) MICCAI 1998. LNCS, vol. 1496, pp. 934-943. Springer, Heidelberg (1998)

3. Su, L.M., et al.: Augmented Reality During Robot-assisted Laparoscopic Partial Nephrectomy: Toward Real-Time 3D-CT to Stereoscopic Video Registration. J. Urology 73(4), 896-900 (2009)

4. Teber, D., et al.: Augmented Reality: A New Tool To Improve Surgical Accuracy during Laparoscopic Partial Nephrectomy? Preliminary In Vitro and In Vivo Results. European Urology 56(2), 332-338 (2009) 
5. Cheung, C.L., et al.: Fusion of stereoscopic video and laparoscopic ultrasound for minimally invasive partial nephrectomy. In: Medical Imaging 2009: Visualization, Image-Guided Procedures, and Modeling. Proc. SPIE, vol. 7261, pp. 726109 726110 (2009)

6. Leven, J., et al.: DaVinci canvas: a telerobotic surgical system with integrated, robot-assisted, laparoscopic ultrasound capability. In: Duncan, J.S., Gerig, G. (eds.) MICCAI 2005. LNCS, vol. 3749, pp. 811-818. Springer, Heidelberg (2005)

7. Linte, C.A., et al.: Virtual reality-enhanced ultrasound guidance: A novel technique for intracardiac interventions. Comput. Aid. Surg. 13, 82-94 (2008)

8. Lindseth, F., Tangen, G., Lango, T., Bang, J.: Probe calibration for freehand 3D ultrasound. Ultrasound Med. Biol. 29(11), 1607-1623 (2003)

9. Mercier, L., Lango, T., Lindseth, F., Collins, D.L.: A review of calibration techniques for freehand 3-D ultrasound systems. Ultrasound Med. Biol. 31(4), 449-471 (2005)

10. Baghani, A., Eskandari, H., Salcudean, S., Rohling, R.: Measurement of viscoelastic properties of tissue mimicking material using longitudinal wave excitation. IEEE Trans. Ultrason. Ferroelectr. Freq. Control 56(7), 1405-1418 (2009)

11. Poon, T.C., Rohling, R.N.: Tracking a 3-D ultrasound probe with constantly visible fiducials. Ultrasound in Medicine \& Biology 33(1), 152-157 (2007)

12. Prager, R.W., Rohling, R.N., Gee, A.H., Berman, L.: Rapid calibration for 3-D freehand ultrasound. Ultrasound Med. Biol. 24, 855-869 (1998) 